\title{
Diversité De La Macrofaune Du Sol Dans Les Parcelles De Reboisement De La Grande Muraille Verte Au Sénégal
}

\author{
Samb Tamsire \\ Institut fondamental d'Afrique noire Cheikh Anta Diop, laboratoire de \\ zoologie des invertébrés terrestres, BP 206 Dakar-Fann, Sénégal, \\ Cissé Ababacar \\ Institut fondamental d'Afrique noire Cheikh Anta Diop, laboratoire de \\ botanique, \\ Ndiaye Abdoulaye Baïla \\ Institut fondamental d'Afrique noire Cheikh Anta Diop, laboratoire de \\ zoologie des invertébrés terrestres
}

doi: 10.19044/esj.2017.v13n15p185 URL:http://dx.doi.org/10.19044/esj.2017.v13n15p185

\begin{abstract}
Objective: To assess the effect of vegetation cover on soil macrofauna, a study focused on the diversity of soil macrofauna inside and outside reforestation plots of the Great Green Wall in Senegal was carried out. Methodology: The sampling of macrofauna was performed according to the method recommended by the TSBF network (Tropical Soil Biology and Fertility). It involves isolating 10 ground plots along a transect of 50 meters chosen at random in each plot and outside of each plot. The macrofauna was sampled between February and July 2016 during the dry season Results: The results show, in order of importance Hymenoptera, Isoptera, Coleoptera, the Spiders, the isopods and springtails are the taxonomic groups of the study area. The density of each of these taxa is larger inside and outside of the plots. The vertical distribution of macrofauna shows that the $0-10 \mathrm{~cm}$ layer is more densely populated than the deeper layers. Conclusion: This study showed a low diversity of soil macrofauna in the area. It also shows that the vegetation cover affects the density of the macrofauna.
\end{abstract}

Keywords: Macrofauna - Diversity - Density

\section{Résumé}

Objectif: Pour évaluer l'effet du couvert végétal sur la macrofaune du sol, une étude portée sur la diversité de la macrofaune du sol à l'intérieur et à l'extérieur des parcelles de reboisement de la grande muraille verte au 
Sénégal a été réalisé. Méthodologie: L'échantillonnage de la macrofaune a été effectué selon la méthode préconisée par le réseau TSBF (Tropical Soil Biology and Fertility). Elle consiste à isoler 10 placettes de sol le long d'un transect de 50 mètres choisi au hasard à l'intérieur et à l'extérieur de chaque parcelle. La macrofaune a été échantillonnée entre février et juillet 2016 pendant la saison sèche. Résultats: Les résultats montrent, par ordre d'importance, que les Hyménoptères, les Isoptères, les Coléoptères, les Aranéides, les Isopodes et les Collemboles constituent les groupes taxonomiques de la zone d'étude. La densité de chacun de ces taxons est plus importante à l'intérieur qu'à l'extérieur des parcelles. La répartition verticale de la macrofaune montre que la couche $0-10 \mathrm{~cm}$ est plus densément peuplée que les couches les plus profondes. Conclusion: Cette étude montre une faible diversité de la macrofaune du sol dans la zone. Elle montre également que la présence ou l'absence de couvert végétal influe sur la densité de cette macrofaune.

Mots-clés : Macrofaune - Diversité - Densité

\section{Introduction}

La macrofaune du sol, composante la plus importante de la faune des sols tropicaux, joue un rôle essentiel dans leur fertilité. Elle remplit au sein des écosystèmes des fonctions essentielles pour le maintien de la qualité des sols (Giller, 1996). Parmi ces fonctions, figurent la dégradation et l'humification des litières mais aussi la régulation des populations de microorganismes responsables des processus de minéralisation et d'humification (Lafleur, 2003 ; Lavelle \& Fragoso, 2000 ; Grassé, 1986 ; Bachelier, 1963). De même, elle modifie la texture et les propriétés physiques des sols dans les horizons supérieurs habités. La biodiversité de la macrofaune du sol dépend de facteurs tels que le comportement social, la capacité de dispersion, l'humidité, le $\mathrm{pH}$ et la texture du sol (GomaTchimbaklesala, 2008).

En zone sahélo-saharienne, le déficit pluviométrique et l'action anthropique ont fortement perturbé le fonctionnement des écosystèmes entrainant une perte importante de la biodiversité. Pour inverser cette tendance, Union africaine a adopté lors de son $8^{\text {ème }}$ sommet en janvier 2007 l'initiative de la Grande Muraille Verte du Sahara dont l'objectif est de freiner l'avancée du désert.

Au Sénégal, l'essentiel des travaux sur la macrofaune du sol a été effectué dans la partie occidentale du pays porte principalement sur l'un des composantes de la macrofaune du sol (les Isoptères). Très peu d'études portant sur la macrofaune du sol ont été réalisé dans le Ferlo Nord du pays. Ces études avaient pour but de décrire ou de mesurer l'impact de différents 
écosystèmes sur la macrofaune du sol. A notre connaissance, aucune étude sur les variations dans l'espace et dans le temps de la macrofaune du sol n'a été réalisé dans les parcelles de reboisement de la Grande Muraille Verte au Sénégal.

Le présent travail a pour objectif d'étudier la variabilité quantitative et qualitative de la macrofaune du sol à l'intérieur et à l'extérieur des parcelles de reboisement de la Grande Muraille Verte. L'hypothèse suivante a été retenue: la composition de la macrofaune du sol diffère entre les parcelles de reboisement et les zones non protégées.

\section{Méthodologie}

\section{Zone d'étude}

L'étude est réalisée dans le Ferlo Nord du Sénégal, dans les parcelles de reboisement de la Grande Muraille Verte situées entre Widou Thiengoly et Téssékéré (figure 1). C'est une zone semi-aride avec une savane sahélienne. Cette partie du Sénégal est présentée comme étant la plus aride du pays (Ndiaye \& Samb, 2012). Dans cette région règne un climat régional sahélien intermédiaire entre les climats saharien et soudanien. En fonction de la pluviométrie, facteur le plus important dans l'individualisation des domaines climatiques, le domaine sahélien est délimité par les isohyètes $250-300 \mathrm{~mm}$ et $500-550 \mathrm{~mm}$. Cette tranche d'eau est précipitée en une seule saison. La végétation du domaine sahélien qui fait la transition entre les végétations saharienne et soudanienne est caractérisée par des espèces ligneuses peu nombreuses souvent rabougries, la plupart épineuses à rameaux souvent étalés en parasol, à feuillage souvent réduit et caduc (Michel et al., 1969).

Dans le cadre de cette étude, deux types d'habitats ont été pris en compte dans le site: l'intérieur des parcelles de reboisement et l'extérieur de ces parcelles. 


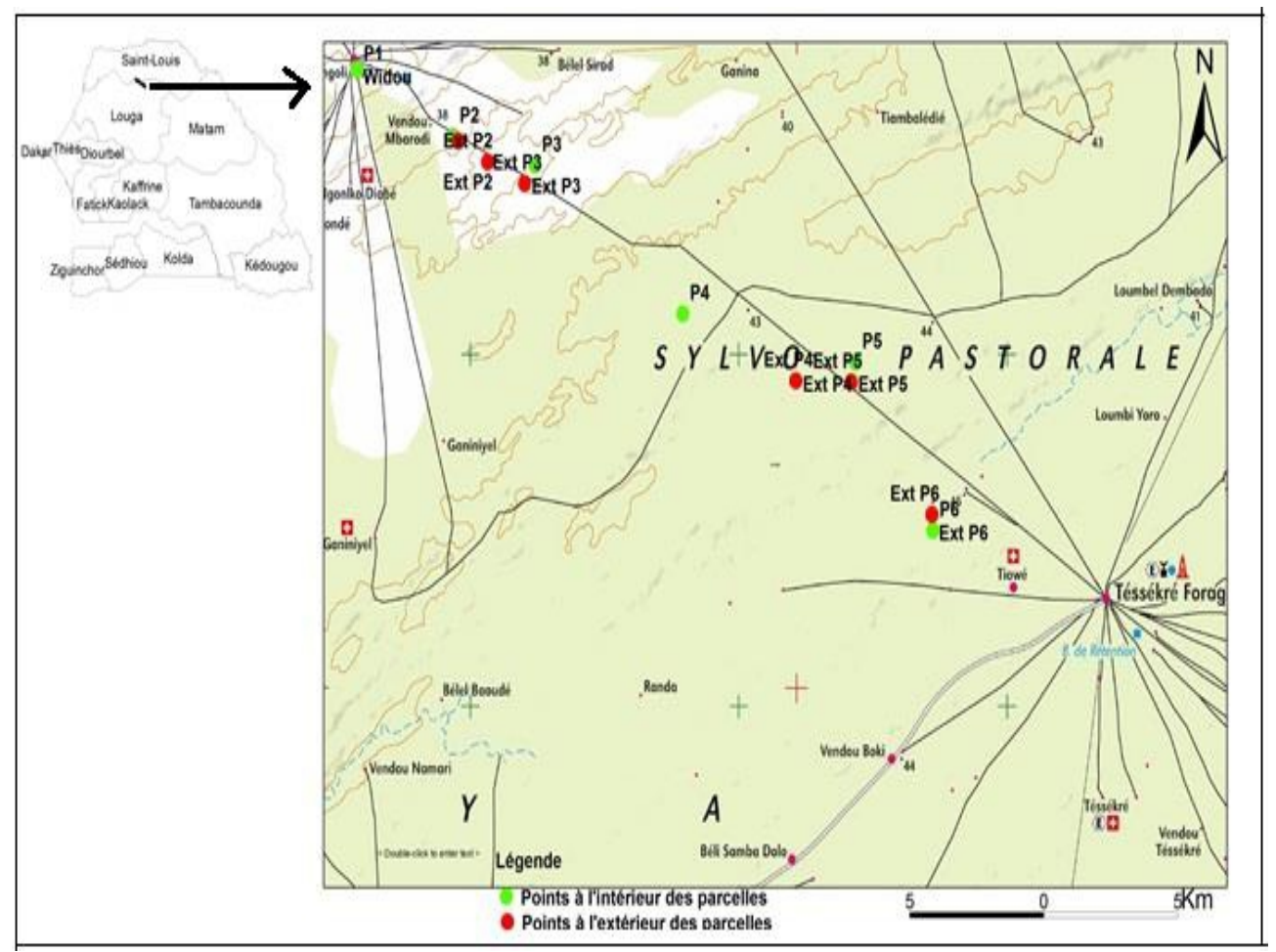

Figure1 : Localisation de la zone d'étude

\section{Méthode d'étude}

La macrofaune a été échantillonnée entre février et juillet 2016 pendant la saison sèche dans chaque type d'habitat du site. L'échantillonnage a été réalisé selon la méthode TSBF (Tropical Soil Biology and Fertility). La méthode consiste à isoler 10 placettes de sol le long d'un transect de 50 mètres choisi au hasard, à l'intérieur et à l'extérieur de chaque parcelle. Les placettes sont distantes de $5 \mathrm{~m}$ et mesurent $25 \mathrm{~cm} \times 25 \mathrm{~cm} \times 30 \mathrm{~cm}$. La litière de la placette est rassemblée et sa faune immédiatement triée à la main. Chaque placette est débitée en trois couches: $0-10 \mathrm{~cm}, 10-20 \mathrm{~cm}$ et $20-30$ $\mathrm{cm}$. Chacune des couches est déposée dans un plateau et les macroinvertébrés triés manuellement.

Les macroinvertébrés visibles à l'œil nu sont prélevés et fixés dans de l'éthanol à $90^{\circ}$. Ils sont ensuite identifiés en fonction du groupe taxonomique (Isopodes, Annélides, Isoptères, Coléoptères, Hyménoptères ou Formicidés). Les individus n'appartenant pas à ces groupes sont classés dans un groupe à part appelé Autres. 


\section{Traitement des données}

\section{Calcul de la densité}

Pour déterminer la densité de la faune (nombre d'individus par $\mathrm{m}^{2}$ ), nous avons procédé à un comptage. La densité est le nombre moyen des individus des différents taxons récoltés au niveau des placettes ramené au mètre carré $\left(\mathrm{m}^{2}\right)$. Le nombre moyen d'individus est calculé en divisant le nombre total des individus du bloc par le nombre de placettes. Cette moyenne est ramenée au mètre carré.

\section{Analyse statistique des données}

Les données ont fait l'objet d'une analyse de variance (ANOVA) et la comparaison des moyennes des densités (Newman-Keuls) ont été effectuées avec le logiciel XLSTAT 6.1 .9 au seuil de $5 \%$ pour évaluer le degré de signification des différences observées.

\section{Résultats} s'agit :

Les différents taxons rencontrés sont notés dans le tableau 1. Il

i) des Chélicérates composés par les Arachnides de l'Ordre des Aranéides (araignées),

ii) des Mandibulates qui comportent des:

- $\quad$ Crustacés terrestres de l'Ordre des Isopodes ;

- Insectes avec les Ordres des Hyménoptères (fourmis) des Isoptères (termites) des Coléoptères et des Collemboles.

Les groupes taxonomiques les plus importants en nombre d'individus sont: les Hyménoptères (Formicidae), les Isoptères, les Coléoptères, et les Aranéides. Les effectifs des Isopodes et des Collemboles sont faibles.

Tableau 1. Effectifs des taxons récoltés à l'intérieur et à l'extérieur des parcelles

\begin{tabular}{ccc}
\hline Taxons & Intérieur parcelles & Extérieur parcelles \\
\hline Hyménoptères (Formicidae) & $1824^{\mathrm{a}}$ & $1127^{\mathrm{a}}$ \\
Isoptères & $483^{\mathrm{a}}$ & $101^{\mathrm{b}}$ \\
Coléoptères & $302^{\mathrm{a}}$ & $97^{\mathrm{b}}$ \\
Aranéides & $48^{\mathrm{a}}$ & $41^{\mathrm{a}}$ \\
Isopodes & $27^{\mathrm{a}}$ & $2^{\mathrm{b}}$ \\
Collemboles & $12^{\mathrm{a}}$ & $1^{\mathrm{b}}$ \\
Autres & $16^{\mathrm{a}}$ & $8^{\mathrm{a}}$ \\
\hline
\end{tabular}

L'analyse de la variance de l'effectif par taxon entre l'intérieur et l'extérieur des parcelles (tableau 1) montre des différences significatives au 
seuil de $5 \%$ pour les Isoptères, les Coléoptères, les Isopodes et les Collemboles.

\section{Densité de la macrofaune du sol}

Les densités moyennes de la macrofaune du sol à l'intérieur et à l'extérieur des parcelles sont respectivement 732,2 ind. $/ \mathrm{m}^{2}$ et 367,2 ind. $/ \mathrm{m}^{2}$. La différence entre les densités moyennes n'est pas statistiquement significative (Test Newman-Keuls, seuil de $5 \%$ ).

Les Formicidés constituent le groupe le plus dense avec 486,40 ind. $/ \mathrm{m}^{2}$ à l'intérieur des parcelles et 300,53 ind. $/ \mathrm{m}^{2}$ en dehors des parcelles. Les densités les plus faibles sont représentées par les collemboles avec 3,2 ind. $/ \mathrm{m}^{2}$ dans les parcelles et 0,26 ind. $/ \mathrm{m}^{2}$ en dehors des parcelles. Les autres groupes taxonomiques constituent des gradients de densité entre les deux extrémités de sorte qu'à l'intérieur des parcelles les Isoptères $>$ Coléoptères $>$ Aranéides $>$ Isopodes $>$ Autres ; qu'en dehors des parcelles les Isoptères $>$ Coléoptères $>$ Aranéides $>$ Autres $>$ Isopodes (tableau 2).

Tableau 2: Densité des macrofaunes du sol par ordre d'importance à l'intérieur et à l'extérieur des parcelles

\begin{tabular}{|c|c|c|}
\hline \multirow{2}{*}{ Ordre d'importance } & \multicolumn{2}{|c|}{ Densité des différents taxons } \\
\cline { 2 - 3 } & Intérieur des parcelles & Extérieur des parcelles \\
\hline \multirow{2}{*}{$1^{\mathrm{er}}$} & Formicidés & Formicidés \\
& 486,40 ind. $/ \mathrm{m}^{2}$ & 300,53 ind. $/ \mathrm{m}^{2}$ \\
\hline \multirow{2}{*}{$2^{\mathrm{e}}$} & Isoptères & Isoptères \\
& 128,80 ind. $/ \mathrm{m}^{2}$ & 26,93 ind. $/ \mathrm{m}^{2}$ \\
\hline \multirow{2}{*}{$3^{\mathrm{e}}$} & Coléoptères & Coléoptères \\
& 80,53 ind. $/ \mathrm{m}^{2}$ & 25,86 ind. $/ \mathrm{m}^{2}$ \\
\hline \multirow{2}{*}{$4^{\mathrm{e}}$} & Aranéides & Aranéides \\
& 12,80 ind. $/ \mathrm{m}^{2}$ & 10,93 ind. $/ \mathrm{m}^{2}$ \\
\hline \multirow{2}{*}{$5^{\mathrm{e}}$} & Isopodes & Autres \\
& 7,20 ind. $/ \mathrm{m}^{2}$ & 128 ind. $/ \mathrm{m}^{2}$ \\
\hline \multirow{2}{*}{$6^{-}$} & Autres & Isopodes \\
& 4,26 ind. $/ \mathrm{m}^{2}$ & 2,13 ind. $/ \mathrm{m}^{2}$ \\
\hline \multirow{2}{*}{$7^{\mathrm{e}}$} & Collemboles & Collemboles \\
& 3,20 ind. $/ \mathrm{m}^{2}$ & 0,26 ind. $/ \mathrm{m}^{2}$ \\
\hline
\end{tabular}

\section{Répartition Verticale de la densité}

En fonction de la profondeur, la distribution de la macrofaune du sol n'est pas homogène. A l'intérieur des parcelles, les couches $0-10 \mathrm{~cm}$ et 10 $20 \mathrm{~cm}$ sont les plus densément peuplées avec, respectivement 65,93\% et $22,57 \%$ de l'effectif total. Tandis qu'en dehors des parcelles, la couche la plus densément peuplée est celle 10-20 cm avec 59,84\% de l'effectif total.

L'analyse de la variance (ANOVA) montre des différences significatives entre les couches $0-10 \mathrm{~cm}, 10-20 \mathrm{~cm}$ et les autres horizons 
(litière et 20-30 cm). Elle montre également que l'effet de la profondeur agit de manière différente sur la répartition verticale des différents groupes taxonomiques; aussi bien à l'intérieur qu'à l'extérieur des parcelles. Les Hyménoptères, les Isoptères et les Coléoptères se trouvent en grand nombre dans la couche 0-10 cm suivie de la couche 10-20 cm avec des différences non significatives. Les Aranéides ont significativement plus d'individus dans les horizons de litière que les autres couches. La distribution des Isopodes, des Collemboles et des Autres macroinvertébrés reste non significatives dans les différentes couches.
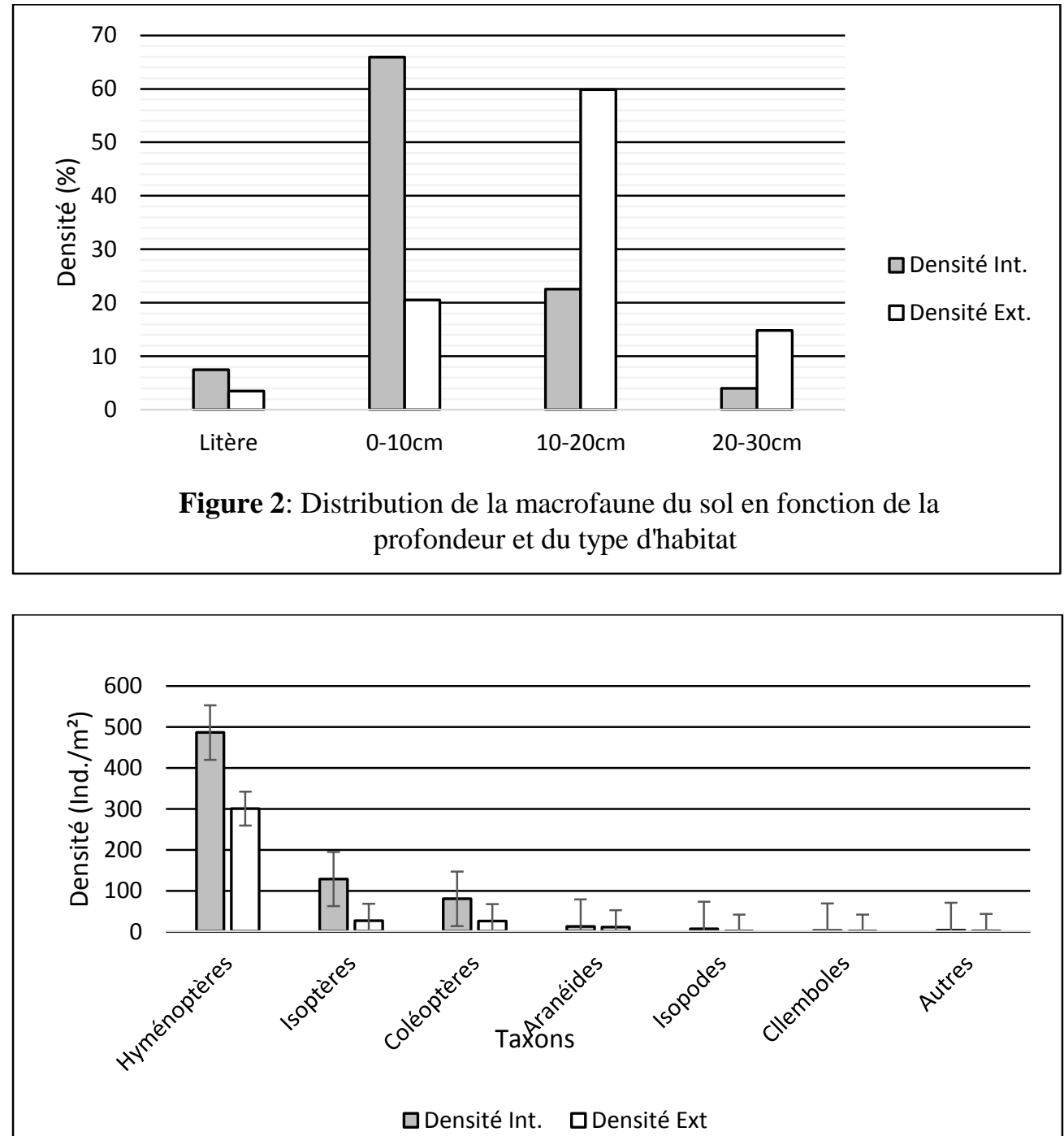

Figure 3: Densité des taxons en fonction du tpyes d'habitat 


\section{Discussion}

Les résultats obtenus montrent que les Hyménoptères et les Isoptères sont les insectes dominants de la macrofaune du sol dans l'écosystème sahélien. Ces résultats s'accordent avec ceux de Samb \& al. (2014) qui ont travaillé dans le Ferlo. L'étude qualitative a permis de recenser une prédominance des fourmis et des termites, ce qui confirme leur qualificatif de macrofaune la plus importante du sol par certains auteurs (Wood \& Sands, 1978, Grassé, 1986, Sarr, 1999).

Les résultats révèlent une faune peu diversifiée du point de vue taxonomique. Cette observation serait liée à la pauvreté du sol en matière organique. Les Arthropodes du sol vivent essentiellement dans la litière dont ils se nourrissent (Chotte et al., 2001). Le nombre de micro arthropodes est en relation avec la teneur en matières organiques. Le sol de la zone d'étude a subi des actions anthropiques (surpâturage, feu de brousse) qui ont des conséquences négatives sur les peuplements de la faune du sol. Comme le notent Fournier et al. (1998): les actions anthropiques comme les pratiques agricoles occasionnent de multiples dommages sur la biodiversité.

La comparaison des densités révèle que les densités les plus importantes sont rencontrées à l'intérieur des parcelles qu'en dehors des parcelles. Ces résultats s'accordent avec ceux trouvés par certains auteurs (Lemvo, 2001 ; Goma-Tchimbaklesala, 2003 et 2008) qui montrent que les densités des termites, des myriapodes et des coléoptères sont supérieures dans les forêts non dégradées que dans les forêts et savanes dégradées.

Selon certains auteurs (Jokimakï, 1998 ; Petit \& Burel, 1998) la structure du paysage peut influencer la répartition des coléoptères et des fourmis en milieu tempéré ou tropical. Les densités de fourmis, de termites et de coléoptères observées au cours de cette étude ne diffèrent pas significativement entre l'intérieur et l'extérieur des parcelles. Ceci est peut être dû à des migrations vers les parcelles suite à une dégradation du couvert végétal en dehors des parcelles.

En accord avec de nombreux auteurs (Montadert, 1985; Mando, 1997; Lemvo, 2001 ; Goma-Tchimbaklesala, 2003 et 2008 ; Samb et al., 2014) nous constatons que la couche $0-10 \mathrm{~cm}$ est plus densément peuplée que les autres couches. La densité de macroinvertébrés observée dans la litière des parcelles s'explique par la présence d'une population plus dense de termites. En effet les termites sont nombreux et actifs pendant la décomposition des litières en saison sèche (Goma-Tchimbakala \& BernhardReversat, 2006). En milieux tropicaux, les processus de recyclage et de stockage de la matière organique et des nutriments se situent principalement dans la litière ou dans les horizons les plus superficiels du sol (Lavelle \& 
Spain, 2001). C'est ainsi que la macrofaune du sol se trouve concentrée dans la litière et la couche $0-10 \mathrm{~cm}$.

L'étude montre que la structure du paysage ou la couverture végétale influe sur la densité et la répartition des macroinvertébrés du sol.

\section{Remerciements}

Nous remercions l'Unité Mixte Internationale ESS 3189 et l'Observatoire Homme Milieux Téssékéré (Labex DRIHM) qui ont financé ce travail.

\section{References:}

1. Bachelier G. 1963. La vie dans les sols. ORSTOM, Paris.

2. Carabidae). Gibier Faune Sauvage, Game Wildl, 15 (Hors série Tome 1): 43-53.

3. Chotte J. L., Duponnois R., Cadet P., Adiko A., Villenave C., Agbogba C. \& Brauman A. 2001. «Jachère et biologie du sol en Afrique tropicale ». In : Floret et Pontanier; eds. La jachère en Afrique tropicale, 2, John Libbey Eurotext, Paris : 85-121.

4. Fournier E., Loreau M. \& Havet P. 1998. Effect of new agricultural management

5. Giller P.S. 1996. The diversity of soil communities, the_pour man_s tropical rainforest'. Biodiversity and conservation ; $5: 135-168$.

6. Goma-Tchimbakala J. 2003. Dynamique de la matière organique et de l'azote en plantations de limba (Terminalia superba Engler et Diels) au Congo: comparaison avec une forêt naturelle. Thèse de Doctorat, Université Paris VI, 180p.

7. Goma-Tchimbakala J. 2008. Diversité de la macrofaune du sol de la forêt de l'ex ORSTOM de Brazzaville (Congo). Annales de l'Université Marien NGOUABI, 2008 ; 9 (4) : 100-108.

8. Goma-Tchimbakala J. and Bernhard-Reversat F. 2006. Comparison of litter dynamics in three plantations of an indigenous timber-tree species (Terminalia superba) and a natural tropical forest in Mayombe, Congo. Forest Ecology and Management; 229: 304-313.

9. Grassé P. 1986. Termitologia, Tome 3, Masson, Paris.

10. Jokimakï J., Huhta E., Itämies J. and Rahko P. 1998. Distribution of arthropods in relation to forest patch size, edge, and stand characteristics. Revue Canadienne de Recherche Forestière; 28: 1068-1072.

11. Lafeur B. 2003. Fourmis et fertilité des sols dans les pessières à lichens. Natur. Canadien 127 (2) : 22-25. 
12. Lavelle P. \& Fragoso C. 2000. La macrofaune du sol : une ressource en danger dans un monde en changement. Rapport du colloque international du 19 au 23 juin 2000, Bondy, 44p.

13. Lavelle P. and Spain A. 2001. Soil Ecology, 1288p

14. Lemvo I. 2001. Étude de la macrofaune du sol dans les plantations de limba (Terminalia superba Engler et Diels) au Mayombe. Mémoire, Institut de Développement Rural, Université Marien Ngouabi, Brazzaville, 50p.

15. Mando A. 1997. Effect of termites and mulch on the physical rehabilitation of structurally crusted soils in the Sahel. Land Degradation and Development 8: 269-278.

16. Michel P. 1969. Le bassin des fleuves Sénégal et Gambie. Etude géomorphologique. Thèse Doctorat ès Lettres, Strasbourg, 1167 p.

17. Montadert M. 1985. Le peuplement de vers de terre et autres macroinvertébrés du sol d'une forêt équatoriale humide (Mayombe). Mémoire de DEA Ecologie, Faculté d'Orsay, Paris, 41p.

18. Ndiaye A. B. \& Samb T. 2012. Les termites (Isoptera) dans les parcelles de reboisement de la grande muraille verte entre WidouThiengoly et Tessekere (Sénégal). Les cahiers de l'Observatoire International «Homme-Milieu » Tessekere, $N^{\circ}$ 1, 63 73.

19. Petit S. and Burel F. 1998. Effects of Landscape dynamics on the metapopulation of a ground beetle (Coléoptèra, carabidae) in a hedgerow network. Agriculture Ecosystems and Environment; 69: 243252.

20. practices on the structure and diversity of ground-beetle communities (Coleoptera,

21. Samb T., Ba CH. T., Ndiaye A. B. 2014. Étude de l'effet des de cannes du petit mil et de la fumure d'ovins et de caprins sur la macrofaune du sol en zone sahélienne (Matam, Sénégal), Bulletin de l'IFAN Ch. A. Diop, sér. A, 2014, 53 (2) : 101-115

22. Sarr M. 1999. Étude écologique des peuplements de Termites dans les jachères et dans les cultures en zone soudano-sahélienne au Sénégal. Th. 3e cycle, UCAD, Dakar, 117 pp.

23. Wood T. G. \& Sands W. A. 1978. "The role of termites in ecosystems". In: Production ecology of ants and termites. Ed. M. V. Brian. Cambridge University Press, Cambridge: 245-292. 